\title{
Rúbrica de evaluación como instrumento mediador de la reflexión en el practicum de maestros de educación infantil
}

\author{
Tatiana López Jiménez*| Rosa María Colomina Álvarez***
}

En el contexto de practicum de maestros de educación infantil, se reportan resultados de un estudio cuyo propósito fue describir y comprender el uso de la rúbrica de evaluación como artefacto mediador de la reflexión conjunta entre maestros de educación infantil en formación y su tutor universitario. Se asume una perspectiva sociocultural y un enfoque interpretativo, desde los cuales se llevó a cabo un estudio de casos múltiples en el que participaron 6 casos, correspondientes a una universidad chilena. Se registraron mediante video 18 sesiones de tutorías individuales que fueron analizadas a partir del modelo de la interactividad. Los resultados muestran, por una parte, que hay diversas formas de organizar las tutorías y, por otra, que no es el instrumento per se lo que determina el uso que puede darse a la rúbrica, sino que éste depende de cómo el tutor decida apoyar las necesidades específicas de cada maestro en el proceso de formación.

The present article reveals the results obtained in a study aimed towards describing and understanding the use of the rubric for assessment as a reflection mediating artifact between early childhood education teachers in training and their university tutor (within the practicum context). Assuming a sociocultural perspective and an interpretive approach we carried out a multiple case study, using 6 cases from a Chilean university. A total of 18 individual tutoring sessions were recorded in video and analyzed using the interactivity model. The results show, on the one hand, that there are different ways of organizing classes and tutorials and, on the other, that it is not the instrument per se which determines the way in which the rubric might be used, but it rather depends on how the tutor decides to tend to the specific needs of each one of the teachers to be during their training process.
Palabras clave

Maestros de educación infantil

Practicum

Proceso de reflexión

Rúbricas de evaluación

Artefactos culturales

Keywords

Early childhood education teachers

Practicum

Reflection process

Rubric for assessment

Cultural artifacts

Recepción: 16 de noviembre de 2019 | Aceptación: 16 de marzo de 2021

DOI: https://doi.org/10.22201/iisue.24486167e.2021.174.59681

* Académica de la Escuela de Pedagogía de la Pontificia Universidad Católica de Valparaíso (Chile). Doctora en Psicología de la Educación. Líneas de investigación: formación de profesores; educación infantil; procesos de reflexión de la práctica pedagógica. Publicación reciente: (2019, en coautoría con M.V. Leiva Guerrero), "Uso de plataforma virtual para retroalimentar la formación del profesorado", Pensamiento Educativo. Revista de Investigación Educacional Latinoamericana, vol.56, núm. 2,pp. 1-17.DOI: https://doi.org/10.7764/PEL.56.2.2019.5.CE: tatiana.lopez@pucv.cl

** Académica del Departamento de Cognición, Desarrollo y Psicología de la Educación de la Universitat de Barcelona (España). Doctora en Psicología de la Educación. Líneas de investigación: análisis de la actividad conjunta; discurso educativo; evaluación de los aprendizajes; influencia de las TIC en la construcción del conocimiento; reflexión sobre situaciones de la práctica para la mejora de la formación de profesorado. Publicación reciente: (2019, en coautoría con M. Clarà, T. Mauri y J. Onrubia), "Supporting Collaborative Reflection in Teacher Education: A case study”, European Journal of Teacher Education, pp. 175-191. DOI: https://doi.org/10.1080/02619768.2019.1576626. CE:rosacolomina@ub.edu 


\section{INTRODUCCIÓN}

La formación de los maestros es uno de los ámbitos fundamentales para la mejora de los sistemas educativos (Darling-Hammond y Lieberman, 2012; Fernández-Fernández et al., 2016; Mena-Marco et al., 2017), más aún cuando el interés es fortalecer la educación infantil, la cual es prioridad en las agendas de política pública de numerosos países (Cobano-Delgado et al., 2019; Pardo y Opazo, 2019). Este interés radica en la abundante evidencia científica multidisciplinaria que ha mostrado los impactos positivos, tanto individuales como sociales, de la educación de la primera infancia (OECD, 2017; Pardo y Opazo, 2019) y, como consecuencia de esta evolución, la creciente necesidad de mejorar la competencia profesional de maestros de educación infantil ${ }^{1}$ durante su formación (Fonsén y UkkonenMikkola, 2019).

Este estudio ${ }^{2}$ busca comprender cómo se utiliza la rúbrica de evaluación durante el practicum como artefacto mediador para permitir a los maestros en formación, en conjunto con su tutor universitario, reflexionar y evaluar el desempeño pedagógico realizado en el aula. La literatura señala que, dadas sus características, el practicum permite que el maestro en formación desarrolle un doble proceso de aprendizaje, tanto en la socialización como en la construcción de conocimiento profesional (Sewell et al., 2017), dado que lo conecta con situaciones reales de enseñanza y aprendizaje que favorecen el proceso de reflexión (Beauchamp, 2015; Kaasila y Lauriala, 2012).

Diversos estudios como los de Gelfuso y Dennis (2014); Korthagen et al. (2001); Mauri et al. (2015; 2016a) y Salinas et al. (2018) establecen que el practicum en la formación de maestros posibilita el análisis del conocimiento de y para la enseñanza, este último identificado como un aspecto central de dicha formación por las investigaciones referidas a educación infantil (Jiménez y Colomina, 2014). De acuerdo con Fernández-Fernández et al. (2016), Gelfuso y Dennis (2014) y Lawson et al. (2015), el conocimiento de y para la enseñanza proviene de las actividades que los maestros en formación realizan: de sus experiencias, de las representaciones que construyen, las decisiones que toman y las reflexiones que las sustentan.

En este sentido, el aprendizaje que desarrolla el maestro durante el practicum se caracteriza por ser auténtico, situado, útil para abordar y resolver problemas reales y dificultades surgidas en la práctica educativa que el docente en formación realiza en el centro escolar (Clarà et al., 2019; Korthagen et al., 2001; Mauri et al., 2015; 2016a). En términos de competencias profesionales, se espera que, durante el practicum, el maestro alcance niveles altos de reflexión (Beauchamp, 2015; Salinas et al., 2018; Schön, 1998) que le permitan resolver las dificultades que presenta la práctica pedagógica (Saiz y Susinos, 2018).

\section{EL PROCESO DE REFLEXIÓN EN EL PRACTICUM DESDE UNA PERSPECTIVA SOCIOCULTURAL}

Estudios empíricos señalan que el proceso de reflexión está en el centro de muchos programas de formación de maestros, en los cuales se enfatiza la relevancia de incorporar durante el practicum experiencias que promuevan este proceso (Clarà et al., 2019; FernándezFernández et al., 2016; Gelfuso y Dennis, 2014; Lawson et al., 2015; Mauri et al., 2016a; Korthagen et al., 2001). A pesar de que la noción de reflexión presenta ambigüedades en cuanto a su conceptualización, naturaleza y características (Salinas et al., 2018; Clarà et al., 2019), la literatura da cuenta de la necesidad

\footnotetext{
${ }^{1}$ En el presente estudio se privilegiará el uso del genérico masculino, que incluye el género femenino. El uso no marcado es lingüísticamente correcto y favorece la economía lingüística, según la RAE (Cf. Diccionario Panhispánico de Dudas).

2 Este estudio fue financiado por la Comisión Nacional Científica y Tecnológica CONICYT- PAI/ INDUSTRIA 72120398. Ministerio de Educación de Chile y su programa Becas Chile.
} 
de continuar investigando cuál es la mejor manera de ayudar a desarrollar este proceso durante el practicum.

Entre las situaciones formativas planificadas para favorecer la reflexión en los programas de formación se encuentra una amplia variedad, entre ellas: trabajos escritos a partir de los cuales se evalúa la reflexión (Salinas et al., 2018), sesiones de tutorías (Chung y Van Es, 2014), análisis de videos (Jiménez y Colomina, 2014), compilaciones de situaciones vivenciadas por los maestros en formación que permitan elaborar reflexiones que posteriormente serán registradas mediante portafolios (Oakley et al., 2014) y programas de apoyo orientados al desarrollo de la reflexión (MenaMarco et al., 2017). Sin embargo, aunque este tipo de situaciones tiene como objetivo que los maestros en formación desarrollen la reflexión (Beauchamp, 2015; Darling-Hammond y Lieberman, 2012), no permiten conocer la influencia que tiene el artefacto mediador para que estos docentes sean capaces de reflexionar.

La literatura destaca a autores como Schön (1998) y Dewey (1989) como pioneros en los estudios de la reflexión sobre "las situaciones de la práctica”, a la que consideran un elemento clave para la mejora de la comprensión sobre la actuación docente. Schön (1998) alude a la importancia de tener como eje fundamental en la formación inicial del profesorado la reflexión en y sobre la acción docente, puesto que sólo es posible formar profesionales reflexivos cuando se trabaja en y sobre la propia actividad profesional. Por su parte, este estudio asume los planteamientos de Dewey sobre la reflexión, entendiéndola como un doble proceso de análisis y síntesis (Dewey, 1989; Mauri et al., 2016a; 2016b). La reflexión es definida como "un proceso de indagación que hace el maestro en formación conjuntamente con su tutor universitario (u otro más experto) en el que abordan los dilemas presentados en el desempeño pedagógico realizado en el aula infantil" (Jiménez y Colomina, 2014: 19); en este proceso la discusión se centra en la identificación de diferentes elementos implicados en la práctica pedagógica, en situaciones imprevistas y complejas; y se busca comprender lo que se hizo, por qué se hizo, cuál era el propósito y cómo podría mejorar su desempeño pedagógico.

En coherencia con lo anterior, se asume una perspectiva sociocultural para comprender el proceso de enseñanza-aprendizaje de los maestros en formación, donde la enseñanza se define en términos de influencia educativa (Coll et al., 2008); es decir, en términos de ayudas prestadas a la actividad constructiva del alumno, en este caso, maestros en formación. La enseñanza, entendida como influencia educativa, refiere a los procesos que usan los profesores, en este estudio, tutores universitarios, para ayudar de forma intencional, sistemática y planificada a los procesos de construcción de significados y de atribución de sentido que los maestros en formación realizan sobre los contenidos objeto de aprendizaje (Coll et al., 2008). El estudio de la influencia educativa tiene una amplia trayectoria, tanto en la educación formal como en la formación inicial y continua del profesorado (Clarà et al., 2019; Mauri et al., 2016a; 2016b), y permite ofrecer un marco explicativo para comprender la reflexión conjunta en el practicum.

Los estudios empíricos centrados en la influencia educativa de los procesos de reflexión durante la formación inicial de los docentes señalan que las ayudas ofrecidas por el tutor universitario en situaciones de reflexión conjunta se articulan mediante el diálogo (Mauri et al., 2016a). Por otra parte, estos estudios señalan que los tutores universitarios apoyan la reflexión conjunta de modo específico, y se centran en aspectos característicos, de manera que ayuden a los maestros en formación a identificar los factores que intervienen en las situaciones de la práctica, y a analizarlos e interpretarlos (Mauri et al., 2015). El trabajo de Mauri et al. (2016b) refiere que, en la actividad entre tutor y maestro en formación (que facilita una reflexión compleja basada en la relación dialéctica entre teoría y práctica), se encuentran 
diferentes ayudas que ofrecen los tutores al maestro en formación, relacionadas con el grado, la distribución y el andamiaje de la ayuda ofrecida por el tutor al maestro en formación.

Por otra parte, Clarà et al. (2019) investigaron situaciones en que los tutores universitarios dialogaban colaborativamente sobre reflexiones escritas registradas por los maestros en formación sobre situaciones experimentadas en el aula. Entre sus resultados señalan que la reflexión colaborativa se organiza en un proceso que contiene una doble dinámica: desde el análisis hasta la síntesis, y desde la facilitación abierta hasta la facilitación directiva de los tutores. Además, en la organización social de la reflexión se identificó una secuencia de cuatro fases: clarificación, exploración, focalización e interpretación de la situación reflexionada.

Las investigaciones precedentes revelan la importancia de diseñar e implementar — durante el practicum de maestros - situaciones de enseñanza y aprendizaje que incorporen procesos de reflexión (Lawson et al., 2015), además de evaluación formativa, para conseguir el ajuste progresivo de las ayudas al aprendizaje, tanto desde la perspectiva de quienes las ofrecen como de quienes las solicitan y las reciben (Coll et al., 2008).

Los estudios recientemente presentados se centran en el proceso de reflexión colaborativa entre el tutor universitario y el maestro en formación; en ellos destaca la existencia de diversas formas de organizar la actividad conjunta, algunas de las cuales aparecen de forma regular durante el periodo estudiado y cumplen una función específica para el aprendizaje de la reflexión en el practicum (Clarà et al., 2019; Mauri et al., 2015, 2016a, 2016b; Jiménez y Colomina, 2014). En las situaciones anteriormente descritas los artefactos mediadores que guiaron la actividad conjunta fueron el diálogo (Mauri et al., 2016a) y las reflexiones escritas de los participantes (Clarà et al., 2019; Mauri et al., 2015). De ahí la necesidad de investigar y comprender cómo determinados artefactos median la actividad conjunta y permiten a los maestros en formación desarrollar procesos de reflexión sobre su propia práctica.

\section{LAS RÚBRICAS DE EVALUACIÓN COMO ARTEFACTOS MEDIADORES EN EL PRACTICUM}

Los estudios precedentes de la influencia educativa en el practicum ofrecen un marco teórico amplio que orienta la formación inicial de los maestros (Clarà et al., 2019; Mauri et al., 2015; 2016a; 2016b). En ellos se concibe al aprendizaje como un proceso que va de lo interpersonal a lo intrapersonal, y al conocimiento como resultado de la interacción de los sujetos en su contexto (Vygotsky, 1979). Estos intercambios sociales entre los individuos están mediados por artefactos culturales que funcionan como eslabones entre lo personal e individual, y lo social y colectivo (Vygotsky, 1979).

En este sentido, Cole (1999) plantea que la cultura comprende un conjunto de artefactos históricos culturales que son socialmente trasmitidos y pueden regular el curso del desarrollo psicológico; los artefactos corresponden a herramientas materiales y simbólicas almacenadas a través del tiempo y trasmitidas a través de procesos sociales. En el contexto de la formación inicial de los maestros, Chung y Van Es (2014) señalan que determinados artefactos (p. ej. video clips, plataformas virtuales, diarios escritos, rúbricas u otros), por su origen social y cultural, juegan un papel intermediario fundamental en la realización de la actividad humana, y pueden actuar como mediadores para apoyar el aprendizaje de los maestros sobre la práctica educativa.

En lo que respecta a las rúbricas de evaluación, éstas se asocian comúnmente a la evaluación formativa de los procesos de aprendizaje de los estudiantes (Raposo y Martínez, 2014), o al desarrollo de las competencias en una determinada materia (Cebrián et al., 2014), en contraposición a la certificación o validación que se da a través de la evaluación sumativa. En este escenario, las rúbricas representan una 
práctica educativa muy significativa en la formación del maestro que favorece, entre otros aspectos, la comunicación entre el tutor y el maestro en formación (Alsina et al., 2017).

En este estudio las rúbricas son consideradas como artefactos que tienen el potencial de mediar el aprendizaje de los maestros, puesto que permiten gestionar el aprendizaje de la reflexión sobre la práctica (Baryla et al., 2012; Cano, 2015). Esto es así dado que el uso de las rúbricas de evaluación -y la transparencia en la evaluación- posibilita un seguimiento de las competencias de los maestros y los ayuda a reducir la ansiedad (Panadero y Johnson, 2013), mejorar el sentido de autoeficacia y apoyar la autorregulación (Cano, 2015), todo lo cual, a su vez, facilita indirectamente un mejor desempeño pedagógico en el centro escolar.

Este tipo particular de artefacto, por sus características, permite a los tutores universitarios realizar un seguimiento y evaluación de las competencias adquiridas por los maestros durante el practicum (Cebrián et al., 2014). La rúbrica se concreta mediante el establecimiento de un conjunto de indicadores que miden el progreso y crean, así, una evaluación más objetiva y consistente a través de la clarificación de los criterios para valorar, en términos específicos, el desempeño en la práctica docente (Gámiz-Sánchez et al., 2015; Raposo y Martínez, 2014). Además, la rúbrica posibilita no sólo la mejora de los aprendizajes, sino también un cambio sostenible en la práctica educativa y se convierte, como indican Conde-Jiménez y Martín-Gutiérrez (2016), en un instrumento que propicia la reflexión de los maestros respecto de su propia práctica. En síntesis, la rúbrica orienta la evaluación y reflexión de la práctica educativa del maestro en formación.

Partiendo de las consideraciones precedentes, el estudio se propuso como objetivo general caracterizar el uso de la rúbrica de evaluación como artefacto mediador en la actividad conjunta entre el tutor universitario y el maestro de educación infantil en formación durante el practicum. En coherencia con dicho objetivo se proponen las siguientes preguntas de investigación: 1) ¿cómo se caracteriza y cómo evoluciona la actividad conjunta orientada a la evaluación y reflexión de la práctica de los maestros de practicum en las que se utilizan rúbricas de evaluación?; 2) ¿qué contenidos formativos se ponen de relieve en la actividad conjunta a lo largo del practicum?; y 3 ) ¿cuáles son las particularidades identificadas en la actividad conjunta que se pudieran relacionar con el uso de la rúbrica de evaluación como artefacto mediador de la actividad?

\section{Metodología}

El estudio es de naturaleza cualitativa basado en el paradigma interpretativo (Erickson, 1989), con estudio de casos múltiple (Stake, 2010). Se focaliza en la particularidad y complejidad de casos singulares que permiten alcanzar un entendimiento profundo de la actividad que realizan los participantes en la interacción. Los datos fueron recogidos mediante registros audiovisuales de la actividad que realizaron tutores universitarios y maestros de educación infantil en las sesiones de tutoría individual.

\section{Participantes Y SITUACIONES OBJETO DE ANÁLISIS}

Los casos fueron seleccionados mediante un muestreo intencional con el propósito de acercarse al objeto de investigación y realizar un estudio en profundidad. Cada caso está compuesto por una pareja conformada por una tutora universitaria y una maestra de educación infantil en formación. Se trata de seis casos en total, constituidos por dos tutoras (tutora A y tutora B) y tres maestras en formación cada una (tutora A, maestras en formación MF_1, MF_2 y MF_3; tutora B, maestras en formación MF_4, MF_5 y MF_6). Para garantizar la confidencialidad de las participantes se solicitó firmar un consentimiento informado previo al proceso de recolección de datos. 
El practicum estudiado se sitúa en el penúltimo semestre de un programa de cuatro años de formación de Maestro de educación infantil, perteneciente a una universidad de Chile. El objetivo del practicum es posibilitar que los maestros integren y apliquen en el centro escolar los conocimientos teórico-prácticos desarrollados en la facultad, para demostrar así las competencias profesionales desarrolladas en las diversas asignaturas contempladas en el programa de formación. Durante el practicum las maestras en formación asisten cotidianamente durante una jornada al centro escolar (5 horas). En el horario alterno, asisten a la facultad a diferentes situaciones de enseñanza y aprendizaje propuestas en el plan de formación.

Para este estudio se seleccionó una de las situaciones de enseñanza y aprendizaje propuestas en el plan de formación del practicum, denominada tutoría individual, con el objetivo de evaluar y reflexionar sobre el desempeño pedagógico de las maestras en formación en el aula infantil. En la tutoría individual participa la diada maestra en formación-tutora universitaria, y se compone de dos momentos: en el primero, la tutora visita in-situ a la maestra en formación en el aula infantil, en tres instancias: al inicio, durante el proceso y al finalizar el practicum; en el segundo momento, la tutora se reúne con la maestra en formación a reflexionar y evaluar de manera conjunta su desempeño pedagógico, utilizando la rúbrica de evaluación como un artefacto mediador.
La rúbrica de evaluación incluye un conjunto de indicadores referidos al desempeño pedagógico mediante los cuales se evalúa el logro de las competencias profesionales de la maestra en formación. Es elaborada por el equipo de tutores de la universidad ${ }^{3}$ y se comparte con las maestras al inicio del practicum. La rúbrica de evaluación considera dos ámbitos, el pedagógico y el personal, y para cada uno se proponen dimensiones con sus respectivos indicadores. Respecto al ámbito pedagógico, las dimensiones corresponden a: preparación para la enseñanza, creación de un ambiente propicio para el aprendizaje, enseñanza para el aprendizaje de todos los estudiantes y responsabilidades profesionales. En el ámbito personal las dimensiones son: puntualidad, presentación personal, interacción con los adultos, interacción con los niños y habilidades sociales.

El procedimiento de recogida de datos se realizó durante todo el proceso de practicum (19 semanas), ya que se registraron en video los momentos implicados en la tutoría individual. En este estudio se presentan los hallazgos identificados en el segundo momento de la tutoría, en el cual la tutora universitaria y la maestra en formación reflexionan y evalúan sobre la práctica realizada en el aula infantil. En la Tabla 1 se presenta el total de los datos analizados en cada caso.

Tabla 1. Resumen datos analizados en cada caso objeto de estudio

\begin{tabular}{lcc}
\hline \multicolumn{1}{c}{ Casos objeto de estudio } & No de sesiones registradas & Tiempo de duración \\
\hline Caso 1 (tutora A y maestra 1) & 3 sesiones & $1: 22: 50$ \\
\hline Caso 2 (tutora A y maestra 2) & 3 sesiones & $1: 40: 47$ \\
\hline Caso 3 (tutora A y maestra 3) & 4 sesiones & $1: 50: 16$ \\
\hline Caso 4 (tutora B y maestra 4) & 3 sesiones & $1: 16: 30$ \\
\hline Caso 5 (tutora B y maestra 5) & 3 sesiones & $0: 35: 05$ \\
\hline Caso 6 (tutora B y maestra 6) & 2 sesiones & $8: 07: 03$ \\
\hline Total de datos registrados & 18 sesiones & \\
\hline
\end{tabular}

Fuente: elaboración propia.

3 Incluye dos marcos de referencia nacionales: 1) dimensiones sobre el desempeño pedagógico de los maestros. Ver https:// www.docentemas.cl (consulta: 16 de octubre de 2019); y 2) marco curricular nacional vigente para educación infantil. 


\section{ANÁLISIS DE LOS DATOS}

Con el fin de caracterizar la actividad conjunta que desarrollaron las participantes, tutora universitaria-maestra en formación, de los seis casos estudiados, en las sesiones de tutoría individual, y comprender su evolución a lo largo del practicum, los datos se analizaron mediante el modelo de la interactividad (Coll et al., 2008), el cual implica la ejecución de tres etapas, a saber:

\section{- Etapa 1. Identificar las distintas formas} de actividad (segmentos de interactividad -SI): corresponde al análisis de fragmentos de la actividad conjunta que representan una unidad analítica básica que otorga una visión global de la estructura de la interactividad, su articulación y evolución a lo largo de las sesiones de tutoría individual. Se analizan las actuaciones que la tutora y la maestra en formación llevan a cabo cuando evalúan y reflexionan sobre el desempeño pedagógico realizado en el aula en el que utilizan las rúbricas de evaluación. La identificación de las actuaciones de ambas durante la sesión define quién puede hacer/decir en un momento dado de la actividad.

- Etapa 2. Elaborar mapas de interactividad (MI): corresponde a la elaboración de representaciones gráficas de la estructura que toma la actividad conjunta. Con los mapas se pretende lograr una panorámica global sobre la actividad que realizan tutora y maestra en formación a lo largo de las diferentes sesiones de tutoría individual.

- Etapa 3. Identificar configuraciones de formas de actividad (configuraciones de segmentos de interactividad-CSI): son agrupaciones de SI que aparecen de forma regular y sistemática en el mismo or- den en las diferentes sesiones de tutoría individual que se desarrollan a lo largo del periodo del practicum. Dichas regularidades indican diferentes funciones en la actividad conjunta realizada por la tutora y la maestra en formación.

El análisis de los datos se realizó con el apoyo del software Atlas.ti, versión 7.0; y el control de la fiabilidad mediante un procedimiento de acuerdo interjueces basado en la concordancia consensuada (Anguera, 1990), en el que participaron dos investigadoras (autoras de este artículo). Se obtuvo un 90 por ciento de acuerdo en la codificación del 100 por ciento de los datos.

\section{Resultados}

Los resultados se presentan organizados de acuerdo con las preguntas de investigación, a partir del análisis realizado en cada caso y en las diferentes sesiones. En cuanto a la pregunta, ¿cómo se caracteriza y cómo evoluciona la actividad conjunta orientada a la evaluación y reflexión de la práctica de las maestras en las que se utilizan rúbricas de evaluación?, el análisis de los seis casos y de las 18 sesiones de tutoría individual muestra que la actividad conjunta se caracteriza por la presencia de 19 segmentos de interactividad (SI) que dan cuenta de las particularidades y especificidad que toma dicha actividad en el proceso de formación de cada maestra.

Cada Si se presenta en la Tabla 2, y remite a patrones de actuación articulados entre lo que dicen y hacen la tutora y la maestra en formación; además, está definido por una finalidad formativa específica que responde a un determinado contenido/tarea que se desarrolla durante el practicum. Cada SI se simboliza con un color. ${ }^{4}$

\footnotetext{
${ }^{4}$ N. del Ed. En la versión impresa de Perfiles Educativos, por ser blanco y negro, será difícil distinguir los segmentos en la gráfica. Recomendamos acudir a la versión web para evitar confusión.
} 
Tabla 2. Total de segmentos de interactividad identificados

\begin{tabular}{|c|c|c|c|}
\hline $\mathrm{N}^{\circ}$ & SI identificados & $\begin{array}{l}\text { Descripción de la actividad conjunta tutor } \\
\text { universitario-maestra en formación }\end{array}$ & Color \\
\hline 1 & $\begin{array}{l}\text { Presentación de la sesión de } \\
\text { devolución }\end{array}$ & $\begin{array}{l}\text { Iniciar la actividad conjunta tutora-maestra en formación ex- } \\
\text { plicitando el sentido en el que se configuran ésta y otras sesiones } \\
\text { planificadas durante el practicum. }\end{array}$ & \\
\hline 2 & $\begin{array}{l}\text { Identificación de aspectos prio- } \\
\text { ritarios de la práctica observada }\end{array}$ & $\begin{array}{l}\text { Centrar la atención en aquellos elementos que presentan mayor } \\
\text { necesidad de apoyo para la maestra en formación. }\end{array}$ & \\
\hline 3 & $\begin{array}{l}\text { Comentarios globales del con- } \\
\text { texto de la práctica observada }\end{array}$ & $\begin{array}{l}\text { Proporcionar información específica del contexto, a la maestra en } \\
\text { formación, tanto del centro escolar como del aula, que beneficien } \\
\text { su actuación futura. }\end{array}$ & \\
\hline 4 & $\begin{array}{l}\text { Autoevaluación de su práctica } \\
\text { pedagógica por parte de la } \\
\text { maestra en formación }\end{array}$ & $\begin{array}{l}\text { Autoevaluar su actuación pedagógica a partir de la implementa- } \\
\text { ción de los contenidos aprendidos en el transcurso de su forma- } \\
\text { ción y la planificación. }\end{array}$ & \\
\hline 5 & $\begin{array}{l}\text { Identificación de aspectos } \\
\text { fuertes y débiles por parte de } \\
\text { la tutora }\end{array}$ & $\begin{array}{l}\text { Informar a la maestra en formación sobre puntos fuertes y débiles } \\
\text { de las actuaciones pedagógicas observadas en el centro escolar, } \\
\text { para que tome decisiones sobre los aspectos a mejorar. }\end{array}$ & \\
\hline 6 & $\begin{array}{l}\text { Propuestas de estrategias de } \\
\text { mejora por parte de la tutora }\end{array}$ & $\begin{array}{l}\text { Sistematizar procedimientos concretos para la mejora de las } \\
\text { acciones pedagógicas observadas en la actuación de la maestra en } \\
\text { formación en el aula. }\end{array}$ & \\
\hline 7 & $\begin{array}{l}\text { Análisis conjunto sobre la prác- } \\
\text { tica pedagógica proponiendo } \\
\text { estrategias de mejora }\end{array}$ & $\begin{array}{l}\text { Identificar y revisar actuaciones pedagógicas específicas que no } \\
\text { dieron un buen resultado, en las que se involucra a los niños y el } \\
\text { contenido planificado, y elaborar una propuesta de mejora que } \\
\text { responda al objetivo planteado. }\end{array}$ & \\
\hline 8 & $\begin{array}{l}\text { Análisis por parte de la tutora } \\
\text { sobre la práctica pedagógica } \\
\text { observada }\end{array}$ & $\begin{array}{l}\text { Informar a la maestra en formación sobre los puntos débiles ob- } \\
\text { servados en su práctica pedagógica, con el fin de que identifique } \\
\text { sus errores y sea consciente de las actuaciones pedagógicas a } \\
\text { mejorar, para lo cual la tutora plantea sugerencias concretas. }\end{array}$ & \\
\hline 9 & $\begin{array}{l}\text { Orientaciones sobre el rol } \\
\text { docente durante el proceso de } \\
\text { formación }\end{array}$ & $\begin{array}{l}\text { Orientar a la maestra en formación en la progresión y mejora de } \\
\text { sus competencias pedagógicas, emocionales-motivacionales, y su } \\
\text { participación en el interior de la comunidad educativa. }\end{array}$ & \\
\hline 10 & $\begin{array}{l}\text { Orientaciones sobre el rol } \\
\text { docente en el ámbito laboral }\end{array}$ & $\begin{array}{l}\text { Orientar el desempeño de las competencias pedagógicas de la } \\
\text { maestra en formación; fortalecer el crecimiento profesional y su } \\
\text { inserción al ámbito laboral. }\end{array}$ & \\
\hline 11 & $\begin{array}{l}\text { Valoración de la práctica peda- } \\
\text { gógica por parte de la mentora }\end{array}$ & $\begin{array}{l}\text { Orientar la actuación pedagógica y el desarrollo profesional } \\
\text { tomando como referencia las necesidades y expectativas de ambos } \\
\text { sistemas educativos (centro escolar y universidad). }\end{array}$ & \\
\hline 12 & $\begin{array}{l}\text { Inserción de la maestra en for- } \\
\text { mación en el centro escolar }\end{array}$ & $\begin{array}{l}\text { Conocer cómo se relacionan y confluyen los sistemas educati- } \\
\text { vos, integrando las exigencias planteadas en la escuela y en la } \\
\text { universidad. }\end{array}$ & \\
\hline 13 & $\begin{array}{l}\text { Identificación de los avances } \\
\text { realizados por la maestra en } \\
\text { formación durante el practicum }\end{array}$ & $\begin{array}{l}\text { Especificar los cambios experimentados en la actuación pe- } \\
\text { dagógica de la maestra en formación que denotan evolución o } \\
\text { transición en las decisiones pedagógicas que han ayudado a la } \\
\text { implementación efectiva del currículo. }\end{array}$ & \\
\hline 14 & $\begin{array}{l}\text { Identificación de los avances de } \\
\text { la maestra en formación a par- } \\
\text { tir de los apoyos de los centros } \\
\text { formativos }\end{array}$ & $\begin{array}{l}\text { Evaluar los apoyos recibidos durante su proceso de práctica, en el } \\
\text { ámbito pedagógico y profesional, a partir de los apoyos entrega- } \\
\text { dos por el centro escolar y la universidad. }\end{array}$ & \\
\hline 15 & $\begin{array}{l}\text { Calificación de los aprendiza- } \\
\text { jes, con apoyo de la rúbrica }\end{array}$ & $\begin{array}{l}\text { Calificar el desempeño de la maestra en formación revelando el } \\
\text { nivel de competencia alcanzado, tanto en el ámbito pedagógico } \\
\text { como en el personal. }\end{array}$ & \\
\hline
\end{tabular}


Tabla 2. Total de segmentos de interactividad identificados

\begin{tabular}{cll}
\hline No & \multicolumn{1}{c}{ SI identificados } & \multicolumn{1}{c}{$\begin{array}{c}\text { Descripción de la actividad conjunta tutor } \\
\text { universitario-maestra en formación }\end{array}$} \\
\hline 16 & $\begin{array}{l}\text { Formalización del análisis } \\
\text { realizado durante la sesión, con } \\
\text { apoyo de rúbrica }\end{array}$ & $\begin{array}{l}\text { Guiar a la maestra en formación para que evalúe su desempeño, } \\
\text { indagando en la coherencia interna entre la preparación de la } \\
\text { enseñanza y el proceso de aprendizaje llevado a cabo con los niños } \\
\text { de su nivel. }\end{array}$ \\
\hline $17 \quad \begin{array}{l}\text { Síntesis sobre los aspectos } \\
\text { fuertes y débiles, con apoyo de } \\
\text { la rúbrica }\end{array}$ & $\begin{array}{l}\text { Registrar en la rúbrica de evaluación las fortalezas y debilidades } \\
\text { comentadas a lo largo de la sesión, enfatizando en aquéllas que } \\
\text { deben ser mejoradas en actuaciones futuras. }\end{array}$ \\
\hline 18 & $\begin{array}{l}\text { Acuerdos sobre los aspec- } \\
\text { tos de mejora de la práctica } \\
\text { pedagógica }\end{array}$ & $\begin{array}{l}\text { Recapitulación centrada en las actuaciones pedagógicas observa- } \\
\text { das, manteniendo la dirección hacia la mejora y optimización de } \\
\text { las prácticas educativas de la maestra en formación. }\end{array}$ \\
\hline 19 & $\begin{array}{l}\text { Acuerdos sobre la gestión del } \\
\text { practicum }\end{array}$ & $\begin{array}{l}\text { Concluir la sesión informando aspectos de gestión pedagógica } \\
\text { y/o académica de la facultad o del trabajo en el centro escolar en el } \\
\text { cual realiza el practicum. }\end{array}$ \\
\hline
\end{tabular}

Fuente: elaboración propia.

Respecto a la evolución de la actividad conjunta en cada caso y en las diferentes sesiones, los resultados muestran que los 19 SI identificados se presentan de forma variable. Dicha evolución se representa de manera gráfica en los mapas de interactividad (MI) en las Fig. 1 y 2 (Fig. 1: casos MF_1, MF_2 y MF_3; Fig. 2: casos MF_4, MF_5 y MF_6). Cada MI muestra una panorámica global que refleja la presencia de los
SI en las diferentes sesiones de tutoría, su organización y evolución durante el proceso formativo llevado a cabo conjuntamente entre la maestra en formación y su tutora universitaria. En su eje horizontal cada MI muestra el número de sesión de la tutoría individual realizada con su respectivo tiempo de duración. Cada SI es representado con un color diferente; expresa la organización temporal de la sesión y señala

Figura 1. Mapa de interactividad del trabajo conjunto de la tutora A con las maestras en formación MF_1, MF_2 y MF_3
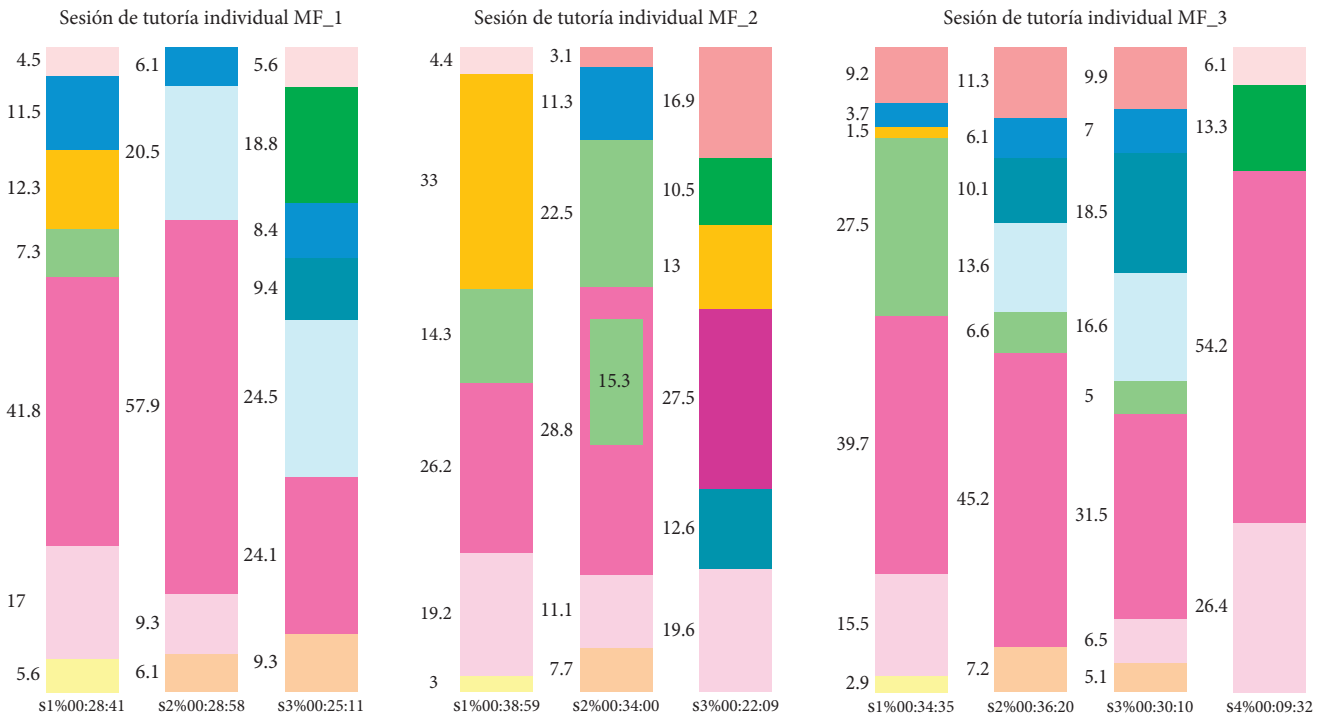

Fuente: elaboración propia. 
Figura 2.Mapa de interactividad del trabajo conjunto de

la tutora B con las maestras en formación MF_4, MF_5 y MF_6
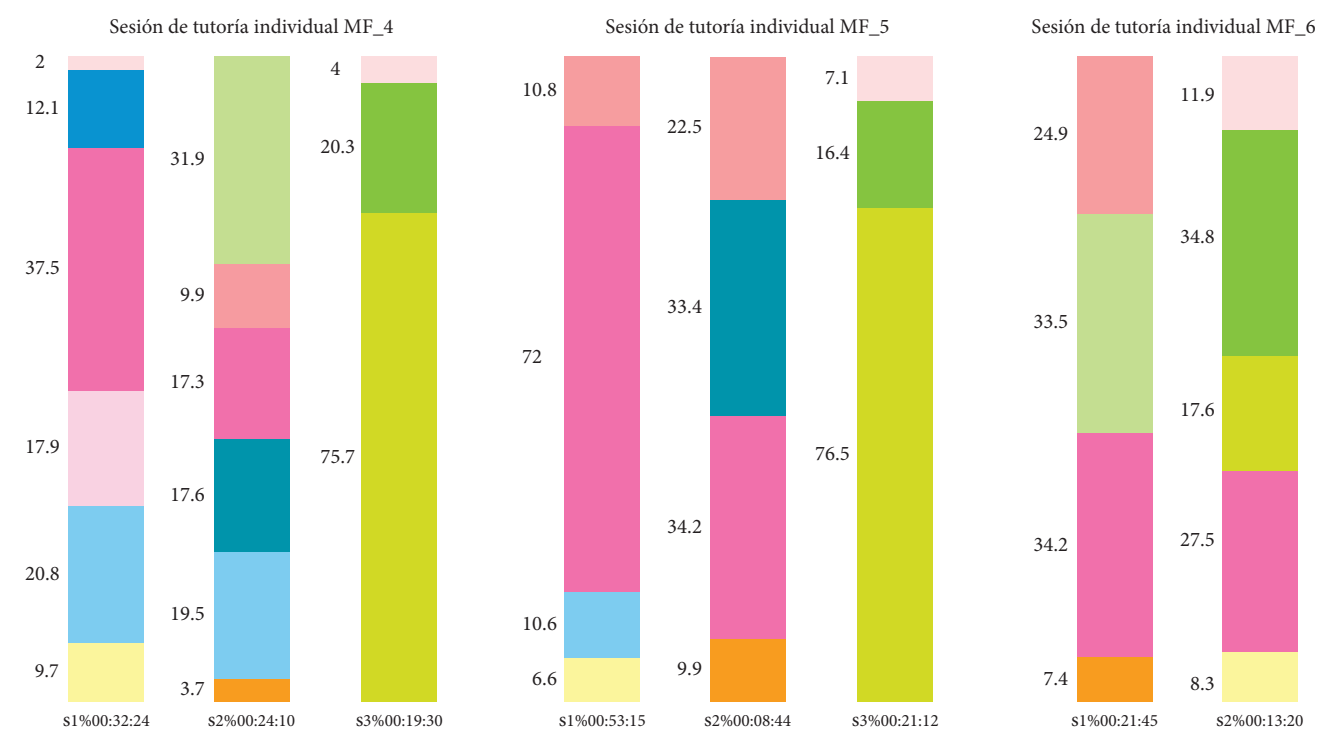

Fuente: elaboración propia.

el porcentaje de tiempo de interacción que se le dedicó a cada segmento. A partir del MI, haciendo una lectura de izquierda a derecha y de abajo hacia arriba, se describió e interpretó el trabajo conjunto realizado por la cada tutora con las maestras en formación en cada sesión de tutoría planificada durante el practicum.

La presencia de los diferentes SI visualizados en los MI (Figs. 1 y 2) da cuenta de la organización temporal de los SI identificados en cada sesión de tutoría. Por una parte, se observa que la presencia de los SI varía en cada caso y en las diferentes sesiones analizadas; por otra parte, se ve que los contenidos formativos implícitos en los SI evolucionan durante el proceso, y que dicha evolución se puede identificar en el punto de vista de los contenidos formativos tratados en las diferentes sesiones o en la participación que tienen las maestras en formación o su respectiva tutora durante la sesión de tutoría individual.

Además, a partir de los MI se puede constatar que, a pesar de que las dos tutoras utilizan el mismo plan formativo, las actividades que desarrollan en cada caso y en las diferentes sesiones son distintas, tanto en el grado de

participación que tienen las tutoras y maestras en formación, como en el contenido formativo que se aborda. Por otra parte, se identifica que cada sesión de tutoría (S1, S2 o S3) presenta una finalidad educativa específica: la primera sesión (S1) se dirigió principalmente a dar comienzo a la sesión y analizar la práctica de la maestra; la segunda sesión (S2) se continuó el análisis de la práctica y, además, se agregó la rúbrica de evaluación; en la tercera sesión (S3) se priorizó orientar el rol docente y el crecimiento profesional, así como cumplimentar la rúbrica de evaluación. Finalmente, a partir de los MI es posible apreciar que cada tutora ajusta su participación a las demandas, necesidades, dificultades o intereses que las maestras en formación presentan, dado que se evidencian cambios en la cantidad de sesiones establecidas en el plan de formación (MF_3 realiza una sesión más S4). Es por ello que, en la evolución de la actividad conjunta, la rúbrica no aparece de manera sistemática en todas las sesiones de todos los casos analizados, sino que se identifican otros tipos de SI relacionados con ámbitos de aprendizajes específicos que se esperan desarrollar en la maestra al término de su formación. 
En cuanto a la segunda pregunta, ¿qué contenidos formativos se ponen de relieve en la actividad conjunta a lo largo del proceso de practicum?, los resultados señalan que los 19 SI identificados tienen la particularidad de centrarse en ámbitos específicos para la formación inicial de maestros. De esta manera, se optó por reorganizar el total de SI tomando como único criterio la finalidad o contenido formativo identificado en el transcurso de las sesiones.

La reorganización de los SI dio como resultado seis tipos: "dar comienzo a la sesión" (reúne SI en que la tutora y maestra en formación inician la sesión); "analizar la práctica de la maestra en formación” (incluye sI valorar la práctica pedagógica y centrar la reflexión en el análisis y mejoras de la actuación en el aula); "orientar el rol docente y crecimiento profesional" (SI referidos a identificar las actuaciones pedagógicas de la maestra que caracterizan su propia manera de enseñar); "guiar su participación en una comunidad de práctica" (SI orientados a guiar a la maestra en la inserción en el centro educativo); "cumplimentar los criterios de evaluación” (SI orientados a garantizar el nivel de desempeño adquirido por la maestra); y "formalizar la finalización de la sesión” (SI referidos a dar término a la sesión).

Cada tipo de SI responde a los contenidos formativos que se espera que la maestra desarrolle durante su proceso de formación. Para los fines de este estudio, en la Tabla 3 sólo se presentan ejemplos de las actuaciones típicas realizadas por la tutora y la maestra en formación en dos tipos de SI que presentan mayor interés. El primer tipo de SI se refiere al "análisis de la práctica de la maestra en formación”, y tiene interés dado que es el único que presenta dos finalidades específicas; por un lado, se identifican SI orientados a valorar la práctica por parte de los diferentes actores implicados en el proceso: autoevaluación de parte de la maestra infantil en formación, y valoración por parte de su tutora universitaria y de la mentora del centro educativo, lo que permite a las participantes establecer un primer grado de negociación de los significados sobre su desempeño en el aula; y, por otro lado, SI centrados en el análisis de la práctica y sus respectivas propuestas de mejora, en los cuales las participantes reelaboran los significados compartidos en relación con la práctica observada y así aseguran una comprensión global de las actuaciones pedagógicas realizadas en el centro escolar con el fin de optimizar su futuro desempeño.

El segundo tipo de SI está orientado a "cumplimentar los criterios de evaluación". Aquí se incluyen los SI cuya finalidad formativa se dirige a calificar el nivel de competencia alcanzado por la maestra, además del SI orientado a formalizar la evaluación de las actuaciones pedagógicas realizada en el aula, y el SI dirigido a sintetizar los aspectos fuertes y débiles observados en la práctica de la maestra en formación. En definitiva, este tipo de SI está orientado a garantizar el rendimiento de la maestra. A partir de la rúbrica se valora, evalúa y califica el nivel de desempeño adquirido durante el periodo observado en el aula y se realiza un seguimiento y registro de las fortalezas y debilidades observadas por la tutora. 


\section{Tabla 3. Agrupación de los tipos de SI identificados}

a partir de la finalidad formativa

\begin{tabular}{|c|c|c|c|}
\hline \multicolumn{2}{|c|}{$\begin{array}{l}\text { Organización de los SI de } \\
\text { acuerdo con su finalidad } \\
\text { formativa }\end{array}$} & Tipos de SI & $\begin{array}{l}\text { Ejemplo de actuación de tutora y maestra } \\
\text { en formación }\end{array}$ \\
\hline \multirow[t]{6}{*}{$\begin{array}{l}\text { Análisis de } \\
\text { la práctica de } \\
\text { la maestra en } \\
\text { formación }\end{array}$} & $\begin{array}{l}\text { Valorar la } \\
\text { práctica } \\
\text { pedagógica }\end{array}$ & $\begin{array}{l}\text { Autoevaluación de su práctica } \\
\text { pedagógica por parte de la } \\
\text { maestra en formación }\end{array}$ & $\begin{array}{l}\text { T: "Me gustaría que tú me dijeras antes de yo } \\
\text { opinar, qué vio usted, cómo se sintió, como } \\
\text { evaluaría su desarrollo con el grupo, cómo te } \\
\text { desenvolviste". } \\
\text { E: "Es que justo en ese momento, cuando usted } \\
\text { llegó los niños se iban al baño para arreglarse, } \\
\text { me puse nerviosa y no hice nada, nada como } \\
\text { educadora” (ej: S1_Caso MF_3). }\end{array}$ \\
\hline & & $\begin{array}{l}\text { Identificación de aspectos } \\
\text { fuertes y débiles por parte de la } \\
\text { tutora }\end{array}$ & $\begin{array}{l}\text { T: "Me gusta iniciar esto siempre con las forta- } \\
\text { lezas, usted tiene muchas fortalezas: tiene muy } \\
\text { pocos niños, tiene buena llegada con ellos, te } \\
\text { reconocen como el adulto que está en la sala..." } \\
\text { (ej:S1_Caso MF_6). }\end{array}$ \\
\hline & & $\begin{array}{l}\text { Valoración de la práctica peda- } \\
\text { gógica por parte de la mentora }\end{array}$ & $\begin{array}{l}\text { T: "Tu educadora me señaló que habías cambia- } \\
\text { do positivamente entre el inicio y ahora. Que } \\
\text { de a poco te estás haciendo cargo del curso, que } \\
\text { al parecer estás sufriendo problemas de salud". } \\
\text { E: "Sí, es que lo que pasa es que duermo mal, } \\
\text { que estoy sobrepasada y me he sentido mal" } \\
\text { (S1_Caso MF_2). }\end{array}$ \\
\hline & $\begin{array}{l}\text { Centrar el aná- } \\
\text { lisis y plantear } \\
\text { propuestas de } \\
\text { mejora }\end{array}$ & $\begin{array}{l}\text { Análisis por parte de la tutora } \\
\text { sobre la práctica pedagógica } \\
\text { observada }\end{array}$ & $\begin{array}{l}\text { T: "La verdad es que yo no vi lo que tú percibes, } \\
\text { yo vi otra cosa. Yo no logré captar motivación o } \\
\text { entusiasmo de parte de los niños y niñas". } \\
\text { E: “ya”(S3_Caso MF_2). }\end{array}$ \\
\hline & & $\begin{array}{l}\text { Propuestas de estrategias de } \\
\text { mejora por parte de la tutora }\end{array}$ & $\begin{array}{l}\text { T: "Como tarea te voy a dejar que tu sala debe } \\
\text { estar ordenada, que debe haber un fichero téc- } \\
\text { nico que cumpla la función de fichero técnico". } \\
\text { E: "sí, entiendo" (S1_Caso MF_4). }\end{array}$ \\
\hline & & $\begin{array}{l}\text { Análisis conjunto sobre la prác- } \\
\text { tica pedagógica y propuestas de } \\
\text { estrategias de mejora }\end{array}$ & $\begin{array}{l}\text { T: "Lo que tú señalaste es súper importante, } \\
\text { porque fuiste capaz de reconocer ciertos } \\
\text { [y]erros que cometiste, no tenías preparada la } \\
\text { música... tampoco los materiales...". } \\
\text { E: "Quisiera señalar algo, yo tenía planificado } \\
\text { hacer otro juego, por eso no lo tenía” (S2_Caso } \\
\text { MF_2). }\end{array}$ \\
\hline
\end{tabular}


Tabla 3. Agrupación de los tipos de SI identificados

a partir de la finalidad formativa

\begin{tabular}{|c|c|c|}
\hline $\begin{array}{l}\text { Organización de los SI de } \\
\text { acuerdo con su finalidad } \\
\text { formativa }\end{array}$ & Tipos de SI & $\begin{array}{c}\text { Ejemplo de actuación de tutora y maestra } \\
\text { en formación }\end{array}$ \\
\hline \multirow[t]{3}{*}{$\begin{array}{l}\text { Cumplimentar la rúbrica de } \\
\text { evaluación }\end{array}$} & $\begin{array}{l}\text { Calificación de los aprendizajes, } \\
\text { con apoyo de la rúbrica }\end{array}$ & $\begin{array}{l}\text { T: "Existe coherencia entre la actividad planifi- } \\
\text { cada y el indicador de evaluación". } \\
\text { E: "Sí, porque yo planifiqué que los niños logra- } \\
\text { ran..." (ej: S2_Caso MF_3). }\end{array}$ \\
\hline & $\begin{array}{l}\text { Formalización del análisis } \\
\text { realizado durante la sesión, con } \\
\text { apoyo de rúbrica }\end{array}$ & $\begin{array}{l}\text { T: "Si tuvieras que hacer algo distinto en tu } \\
\text { experiencia, ¿qué harías de distinto?". } \\
\text { E: "Quizás probar otros materiales, quizás tra- } \\
\text { bajar un poco más la relajación antes de iniciar } \\
\text { la experiencia" (ej: S3_Caso MF_2). }\end{array}$ \\
\hline & $\begin{array}{l}\text { Síntesis sobre los aspectos } \\
\text { fuertes y débiles, con apoyo de } \\
\text { la rúbrica }\end{array}$ & $\begin{array}{l}\text { T: "Tu problema general es que busque solucio- } \\
\text { nes rápidas para trabajar en el aula, que tenga } \\
\text { los mínimos elementos que ayuden a organizar } \\
\text { mejor el espacio". } \\
\text { E: "Sí lo voy [a] hacer pronto. Yo quiero una } \\
\text { sala ordenada y atractiva para los niños" (ej: } \\
\text { S2_Caso MF_6). }\end{array}$ \\
\hline
\end{tabular}

Fuente: elaboración propia.

Respecto a la última pregunta: ¿cuáles son las particularidades identificadas en la actividad conjunta que pudieran relacionarse con el uso de la rúbrica de evaluación como artefacto mediador de la actividad?, a partir de la identificación de los MI de los seis casos analizados se observa que estas particularidades se relacionan con las configuraciones de segmentos de interactividad (CSI). Las CSI comprenden un conjunto de diferentes SI que presentan una sistematicidad en el orden y frecuencia en que aparecen en las diversas sesiones de tutoría individual y en los distintos casos durante el practicum.

La primera CSI orientada al análisis de la práctica se presenta en la Fig. 3. Se identificó en

Figura 3. CSI dirigida al análisis de la práctica entre tutora y maestra en formación

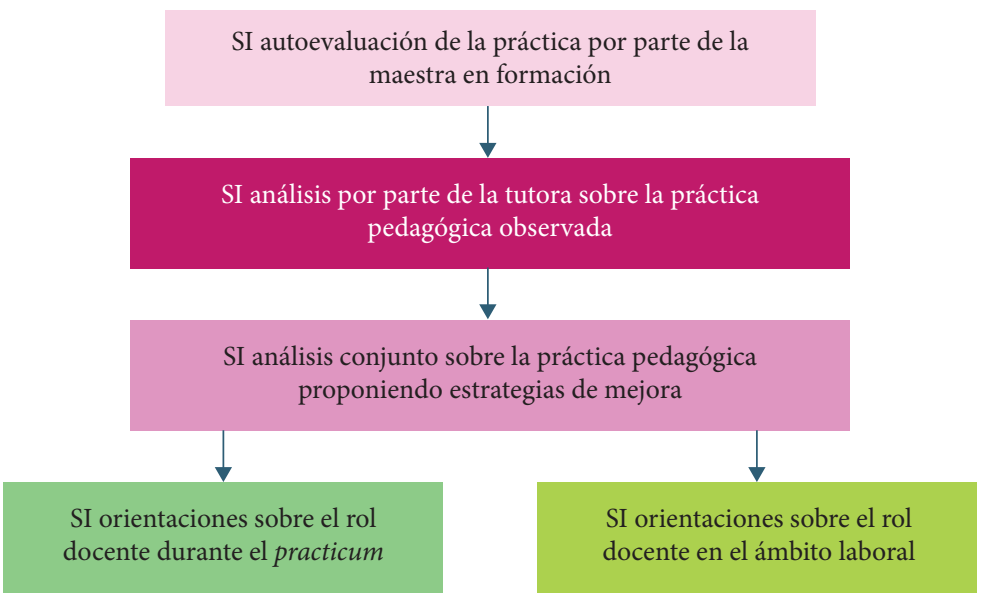

Fuente: elaboración propia. 
Figura 4. CSI dirigida al uso de la rúbrica de evaluación

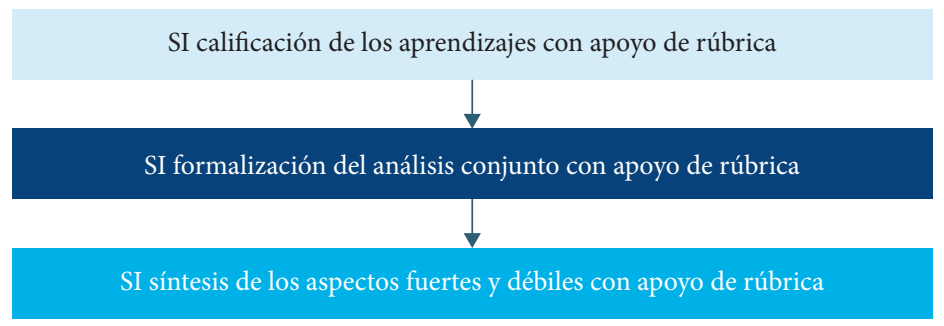

Fuente: elaboración propia.

los MI de los casos MF_1, MF_2, MF_3 y MF_4. Los SI que se mantuvieron de manera sistemática y secuencial en las diferentes sesiones de tutorías analizadas están dirigidos a valorar la práctica pedagógica, realizar un análisis conjunto y plantear propuestas de mejora. Además, en los casos MF_2 y MF_3 (Fig. 1) se incluye la presencia de un cuarto SI orientado al rol docente en el ámbito laboral durante el practicum, el cual evoluciona en el transcurso de las sesiones, p. ej.: Fig. 1. S3 caso MF_2 y S4 caso MF_3). Dicha evolución se explica por la naturaleza formativa de las sesiones analizadas, en que la tutora enfatiza aspectos distintos de acuerdo con las necesidades y requerimientos de las maestras en formación.

La segunda CSI orientada al uso de la rúbrica de evaluación se presenta en la Fig. 4. Dicha CSI se muestra en los MI de los casos MF_1 y MF_3, e involucra los tipos de SI orientados a "cumplimentar los criterios de evaluación” de la rúbrica de evaluación. Incluye los SI de calificación de los aprendizajes, formalización del análisis conjunto y síntesis de los aspectos fuertes y débiles identificados en la actuación pedagógica de la maestra en formación en el aula infantil. En ambos casos la CSI se identifica tanto en las segundas (S2) como en las terceras (S3) sesiones desarrolladas durante el practicum, pero con mayor predominio en estas últimas.

La tercera y última CSI, "rol docente y crecimiento profesional”, se presenta en la Fig. 5. Se identificó en el MI de los casos MF_4, MF_5 y MF_6. Reúne dos tipos de SI: uno orientado al rol docente y crecimiento profesional, y otro dirigido a la incorporación en una comunidad de prácticas. La presencia de esta CSI es característica de las últimas sesiones (S3) de análisis conjunto en los casos que dirige la tutora $\mathrm{B}$, en las que dedica más de la mitad del tiempo de interacción a orientar a la maestra en formación a identificar sus avances en la práctica pedagógica y ayudarla a que proyecte su actuación pedagógica más allá del proceso formativo.

Figura 5. CSI dirigida al rol docente y crecimiento profesional

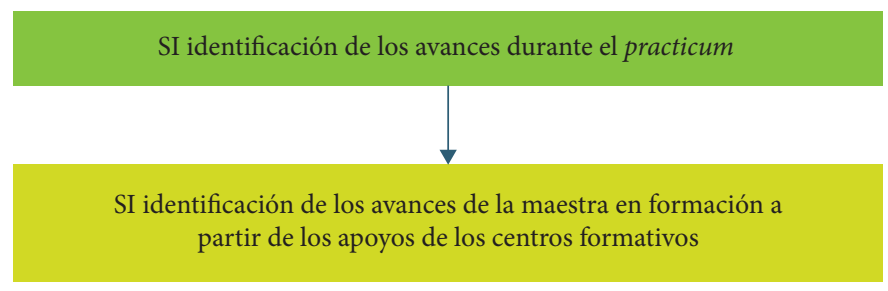

Fuente: elaboración propia.

\section{CONCLUSIONES Y DISCUSIONES}

El análisis de los seis casos de estudio durante el practicum permitió identificar que la actividad conjunta se caracterizó por la presencia de 19 segmentos de interactividad (SI). La evolución de estos SI muestra que la organización de la actividad y el uso de la 
rúbrica orientada a la evaluación y reflexión de la práctica son distintos en cada caso, de modo que no están predeterminados, sino que se construyen en la interacción (Coll et al., 2008). No es el instrumento per se lo que determina el uso que puede hacerse de él, sino que depende de cómo la tutora decide apoyar las necesidades específicas de cada maestra en el proceso de formación.

En cuanto a las finalidades formativas reveladas en los SI, éstas se reorganizaron en seis tipos de SI orientados a: dar comienzo a la sesión, analizar la práctica de la maestra en formación, rol docente y crecimiento profesional, incorporación en la comunidad de prácticas, cumplimentar la rúbrica de evaluación y finalización de la sesión. La presencia de estas finalidades en la temporalidad del practicum permitió identificar el objetivo específico de las distintas sesiones de tutoría (S1, S2 y S3), a partir de las cuales se apoyó de manera diferenciada el proceso de reflexión y evaluación de la práctica de las seis maestras en formación.

Por otra parte, se concluye que las finalidades formativas tuvieron lugar gracias a las ayudas de las tutoras, quienes ponen de relieve aspectos formativos que consideran que las maestras en formación deben saber o reforzar durante el practicum. Lo anterior es coherente con los estudios de Saiz y Susinos (2018), en que los tutores ayudan a los maestros en formación a entender lo que saben y a generar nuevas comprensiones acerca de su práctica pedagógica en el aula.

En cuanto a las particularidades identificadas en la actividad conjunta relacionadas con el uso de la rúbrica de evaluación como artefacto mediador de la actividad, destacamos dos hallazgos relevantes que refieren a la presencia sistemática de algunos SI en el total de los casos analizados. El primer hallazgo refiere a la CSI orientada al análisis de la práctica, en la que se presentan SI orientados a la autoevaluación de la práctica pedagógica por parte de la maestra en formación; luego, el análisis por parte de la tutora sobre la práctica observada; posteriormente se avanza hacia un análisis conjunto, donde discuten los dilemas que se presentaron en la actuaciones pedagógicas y se proponen alternativas de mejora; el proceso culmina con la proyección de la actuación de la maestra en formación y el desarrollo de sus competencias durante el practicum y/o en su inserción laboral. Dicha sistematicidad es coherente con los procesos de reflexión informados en otras investigaciones (Clarà et al., 2019; Jiménez y Colomina, 2014; Mauri et al., 2015, 2016a, 2016b) en las que se señala que no existe una única secuencia para definir un proceso de reflexión conjunta sino que, por el contrario, el proceso de reflexión está supeditado al uso de los artefactos que median la actividad, en este caso el uso que las tutoras otorgan a la rúbrica de evaluación, y a la focalización en distintos aspectos que favorecen la reflexión y evaluación de la práctica de la maestra en formación.

Por otro lado, la presencia de esta CSI orientada al análisis de la práctica plantea algunas reflexiones en relación con lo propuesto por Gelfuso y Dennis (2014), quienes expresan que el proceso de reflexión implica diferentes "patrones regulares" que son más o menos flexibles. En consecuencia, se interpreta que la presencia de la CSI tiene relación con la escala reflexiva propuesta por Alsina et al. (2017) y el modelo ALACT de Korthagen et al. (2001). Aunque estos trabajos están centrados en lo que realiza el aprendiz, a diferencia de este estudio, en el cual nos centramos en la actividad conjunta entre tutora y maestra en formación, es evidente que hay una coincidencia que se explica a partir de la estructura interna de la CSI. En nuestros resultados, la CSI se construye en la interacción: los SI se identifican y se ajustan en función de las necesidades de la maestra en formación, y su presencia permite progresar en el aprendizaje desde la inserción del proceso de practicum hasta su proyección laboral. Lo anterior aporta una mirada distinta a los estudios previos, mismos que destacan la necesidad de identificar, mediante la voz de 
los maestros, la valoración de sus actuaciones pedagógicas en el aula (Salinas et al., 2018).

El segundo hallazgo refiere a la CSI orientada al uso de la rúbrica de evaluación, presentada de manera regular y sistemática en dos de los seis casos de estudios. La estructura de la CSI involucró SI orientados a la calificación de los aprendizajes, la formalización del análisis conjunto y la síntesis de los aspectos fuertes y débiles apoyándose en la rúbrica de evaluación. Debe subrayarse que la rúbrica tiene la ventaja de posibilitar la orientación y evaluación de y para la práctica educativa, lo cual es coherente con lo planteado por Raposo y Martínez (2014).

Además, la presencia de dicha CSI confirma lo señalado por Cano (2015), puesto que las rúbricas posibilitan un tipo de interacción que ayuda al proceso de reflexión de la práctica de los maestros en formación en el cual el tutor ajusta el grado de participación que asume. Por otra parte, la CSI permitió comprender que ésta se relaciona con el ajuste de la ayuda otorgada por las tutoras, lo cual favorece de modo diferente la reflexión y evaluación de la práctica de la maestra. De acuerdo con Clarà et al. (2019) y Mauri et al. (2015; 2016a), esto tiene explicación desde los ámbitos de la práctica que merecen mayor atención, por lo que, aunque la naturaleza de la sesión sea calificar el desempeño de la estudiante, hay ocasiones en que el tutor orienta otros contenidos formativos que surgen en la actuación pedagógica de la maestra en formación.

En cuanto al uso de la rúbrica de evaluación como artefacto mediador, los resultados muestran que su presencia varía en cada caso. En los casos que acompaña la tutora A, la rúbrica cumple dos finalidades complementarias: por un lado, permite profundizar en el aprendizaje del análisis de la práctica, además de compartir con claridad el grado de consecución de los aprendizajes esperados por la maestra en formación, y desarrolla una función tanto formativa como acreditativa de la evaluación. Por su parte, en los casos analizados de la tutora $\mathrm{B}$ los resultados muestran que la rúbrica es utilizada puntualmente, y sólo ocasionalmente se integra al apoyo del aprendizaje; prevalece, por tanto, una función formativa de la evaluación. Esta tutora realiza un seguimiento de la actuación, pero no una calificación. Ella formaliza el análisis al registrar fortalezas y debilidades, y otorgar sugerencias de mejora, lo cual es coherente con CondeJiménez y Martín-Gutiérrez (2016) en cuyo estudio la rúbrica permite tomar conciencia de las dimensiones y aspectos que los maestros noveles deben mejorar para progresar en su aprendizaje. En este estudio dicho aspecto aparece mediado por el rol que cumple la rúbrica en la sesión de tutoría individual.

En cuanto al rol de artefactos mediadores que presenta la rúbrica para propiciar la reflexión de las maestras en formación, los resultados muestran que es posible sistematizar y recopilar información sobre la indagación que realizan conjuntamente tutora y maestra en formación cuando abordan el desempeño pedagógico; además, permite centrarse en los dilemas que se presentan en la actuación pedagógica en el aula, en las situaciones imprevistas y en las mejoras que podría incorporar la maestra en su desempeño futuro. Este hecho permite concluir que la rúbrica de evaluación actúa como mediador de la reflexión, además de que se vincula con los tres aspectos esenciales propuestos por Panadero y Jonsson (2013), en el sentido de que permite: i) clarificar las exigencias de la tarea y los criterios de evaluación; ii) facilitar dar feedback a los maestros en formación; y iii) mejorar el sentido de autoeficacia y apoyarlos en la elaboración de una representación realista de sus propios logros. Por otra parte, se evidencia el rol de la rúbrica en los procesos de autoevaluación, tal como señalan Cano (2015) y Conde-Jiménez y MartínGutiérrez (2016), quienes destacan el valor de la autoevaluación de los aprendizajes adquiridos por los propios maestros en formación.

Finalmente, este estudio aporta nuevos elementos para la comprensión del uso de los artefactos culturales como mediadores de los 
procesos de reflexión en la formación inicial de los maestros en educación infantil, puesto que permiten explicar las formas que toma la actividad conjunta que se realiza en la interacción diádica sobre una situación que puede ser habitual en otros procesos de practicum; por otra parte, sostiene que existe una evolución en los significados compartidos entre los participantes a medida que la actividad conjunta progresa. También, se destaca que el papel del artefacto mediador no viene determinado por él mismo, sino que depende del uso que se le otorgue en la actividad conjunta, y ello depende tanto del maestro en formación como del tutor, que es quien define qué aspecto a evaluar, cuándo y en qué momento de la actividad. Lo anterior es clave para la formación de maestros de

\section{REFERENCIAS}

Alsina, Ángel, Sara Ayllón, Jordi Colomer, Rosario Fernandez-Peña, Judit Fullana, Maria Pallisera, Marc Péez-Burriel y Laura Serra (2017), "Improving and Evaluating Reflective Narratives: A rubric for higher education students", Teaching and Teacher Education, vol. 63, pp. 148-158.

Anguera, María Teresa (1990), "Metodología observacional", en Jaume Arnau, María Teresa Anguera y Juana Gómez (eds.), Metodología de la investigación en ciencias del comportamiento, Murcia, Universidad de Murcia, pp. 125-238.

BARYLA, Ed, Gary Shelley y Trainor William (2012), "Transforming Rubrics Using Factor Analysis", Practical Assessment, Research \& Evaluation, vol. 17, núm. 4, pp. 1-7. DOI: https:// doi.org/10.7275/5nt2-g458

Beauchamp, Catherine (2015), "Reflection in Teacher Education: Issues emerging from a review of current literature", Reflective Practice, vol. 16, núm. 1, pp. 123-141.

CAno, Elena (2015), "Las rúbricas como instrumento de evaluación de competencias en educación superior: ¿uso o abuso?”, Profesorado. Revista de Currículum y Formación de Profesorado, vol. 19, núm. 2, pp. 265-280, en: http://www. ugr.es/ recfpro/rev192COL2.pdf (consulta: 20 septiembre de 2019).

Cebrián, Manuel, José Serrano y Mayerly Ruiz (2014), "Las eRúbricas en la evaluación cooperativa del aprendizaje en la universidad", Comunicar, vol. 43, núm, 22, pp. 153-191. DOI: https://doi.org/10.3916/c43-2014-15 educación infantil, dado que hay escasa evidencia empírica en este ámbito, y al mismo tiempo señala la necesidad imperiosa de fortalecer las competencias profesionales de los maestros de ese nivel educativo (Pardo y Opazo, 2019).

En suma, las aportaciones de este estudio permiten comprender que la rúbrica ayuda a que los maestros en formación y el tutor estén representados en la tarea conjuntay realicen un seguimiento, valoración y mejora del desempeño pedagógico de la maestra. Este tipo de indagaciones nos permite vislumbrar las amplias oportunidades e interrelaciones que los distintos artefactos ofrecen para orientar el proceso de reflexión sobre la práctica pedagógica que realiza el maestro en formación durante el practicum.

ClarÀ, Marc, Teresa Mauri, Rosa Colomina y Javier Onrubia (2019), "Supporting Collaborative Reflection in Teacher Education: A case study", European Journal of Teacher Education, vol. 42, núm. 2, pp. 175-191. DOI: https:// doi.org/10.1080/02619768.2019.1576626

СoвAno-Delgado, Verónica, Vicente Llorent-Bedmar y Alicia Sianes-Bautista (2019), "Formación inicial de profesionales de educación infantil en Alemania y Suecia", Perfiles Educativos, vol. 41, núm. 164, pp. 47-64. DOI: https://doi. org/10.22201/iisue.24486167e.2019.164.59018

Coll, César, Javier Onrubia y Teresa Mauri (2008), "Ayudar a aprender en contextos educativos: el ejercicio de la influencia educativa y el análisis de la enseñanza”, Revista de Educación, núm. 346, pp. 33-70.

Cole, Michael (1999), Psicología cultural: una disciplina del pasado y del futuro, Madrid, Morata.

Conde-Jiménez, Jesús y Ángela Martín-Gutiérrez (2016), "Potencialidades y necesidades de mejora en la formación de profesores noveles universitarios", Revista Electrónica de Investigación Educativa, vol. 18, núm 1, pp. 140-152, en:http://redie.uabc.mx/redie/article/view/767 (consulta: 10 de septiembre de 2019).

Chung, Huy y Elizabeth van Es (2014), "Pre-Service Teachers' Use of Tools to Systematically Analyze Teaching and Learning", Teachers and Teaching, vol. 20, núm 2, pp. 113-135. DOI: https://doi.org/10.1080/13540602.2013.848567 
DARLING-Hammond, Linda y Ann Lieberman (2012), Teacher Education around the World: Changing policies and practices, Nueva York, Routledge.

Dewey, John (1989), Cómo pensamos. La relación entre pensamiento reflexivo y proceso educativo, Barcelona, Paidós.

ERICKson, Frederick (1989), "Métodos cualitativos de investigación sobre la enseñanza”, en Merlin C. Wittrock (ed.), La investigación de la enseñanza II. Métodos cualitativos y de observación, Barcelona, Paidós, pp. 195-301.

Fernández-Fernández, Samuel, José-Miguel AriasBlanco, Rubén Fernández-Alonso, Joaquín Burguera-Condon y Marcelino FernándezRaigoso (2016), "Pensamiento reflexivo e investigador en educación. Aspectos a tener en cuenta en la formación del profesorado", RELIEVE, vol. 22, núm. 2, pp. 1-17. DOI: https://doi.org/10.7203/relieve.22.2.8425

GÁmIz-Sánchez, Vanesa, Norma Torres-Hernández y María Jesús Gallego-Arrufat (2015), “Construcción colaborativa de una e-rúbrica para la autoevaluación formativa en estudios universitarios de pedagogia", REDU: Revista de Docencia Universitaria, vol. 13, núm. 1, pp. 319-338.

Gelfuso, Andrea y Danielle V. Dennis (2014), "Getting Reflection off the Page: The challenges of developing support structures for preservice teacher reflection", Teaching and Teacher Education, vol. 38, núm. 1, pp. 1-11.

KaAsILA, Raimo y Anneli Lauriala (2012), "How do Pre-Service Teachers' Reflective Processes Differ in Relation to Different Contexts?", European Journal of Teacher Education, vol. 35, núm. 1, pp. 77-89. DOI: https://doi.org/10.1080 /02619768.2011.633992

Korthagen, Fred, Jos Kessels, Bob Koster, Bram Lagerwerf y Theo Wubbels (2001), Linking Practice and Theory: The Pedagogy of realistic teacher education, Nueva York, Lawrence Erlbaum Associates.

LAwson, Tony, Melek Çakmak, Müge Gündüz y Hugh Busher (2015), "Research on Teaching Practicum -a Systematic Review”, European Journal of Teacher Education, vol. 38, núm. 3, pp. 392-407. DOI: https://doi.org/10.1080/0261 9768.2014.994060

López Jiménez, Tatiana Maribel y Rosa Colomina (2014), "Los clips de vídeo como medio para ayudar al análisis de las intervenciones docentes de los estudiantes de prácticas”, en Maria Assunçáo Flores y Clara Coutinho (coords.), Formaçao e Trabalho Docente. Diversidade e Convergências, vol. 2, Portugal, De Facto Editores, pp. 17-32.

Mauri, Teresa, Marc Clarà, Rosa Colomina y Javier Onrubia (2015), "Naturaleza de la interacción en procesos de reflexión conjunta sobre situaciones de la práctica por estudiantes de maestro", Papeles de Trabajo sobre Cultura, Educación y Desarrollo Humano, vol. 11, núm. 2, pp. 105-109.

Mauri, Teresa, Marc Clarà, Rosa Colomina y Javier Onrubia (2016a), "Educational Assistance to Improve Reflective Practice among Student Teachers", Electronic Journal of Research in Educational Psychology, vol. 14, núm. 2, pp. 287-309. DOI: https://doi.org/10.14204/ ejrep. 39.15070

Mauri, Teresa, Agurtzane Martínez, Nerea Aguirre, Elena López de Arana, Rosa Colomina, Javier Onrubia y Miguel J. Bascón (2016b), "Construyendo relaciones entre la teoría y práctica. Análisis de ayudas del tutor para favorecer la reflexión y construcción del conocimiento", ponencia presentada en el VIII Congreso Internacional de Psicología y Educación (CIPE), Alicante, junio de 2016.

MenA-Marco, Juanjo, Paul Hennissen y John Loughran (2017), "Developing Pre-Service Teachers' Professional Knowledge of Teaching: The influence of mentoring", Teaching and Teacher Education, vol. 66, pp. 47-59. DOI: https://doi.org/10.1016/j.tate.2017.03.024

OAKLey, Grace, Mark Pegrum y Shannon Johnston (2014), "Introducing e-Portfolios to Pre-service Teachers as Tools for Refection and Growth: Lessons learnt", Asia-Pacific Journal of Teacher Education, vol. 42, núm. 1, pp. 36-50.

OECD (2017), Starting Strong 2017. Key OECD Indicators on Early Childhood Education and Care. Starting strong, París, OECD Publishing. DOI: https://doi.org/10.1787/9789264276116-en

Panadero, Ernesto y Anders Jonsson (2013), “The Use of Scoring Rubrics for Formative Assessment Purposes Revisited: A review", Educational Research Review, vol. 9, pp. 129-144. DOI: https://doi.org/10.1016/j.edurev.2013.01.002

PArdo, Marcela y María-José Opazo (2019), "Resistiendo la escolarización desde el aula. Explorando la identidad profesional de las docentes de primera infancia en Chile", Cultura y Educación, vol. 31, núm. 1, pp. 67-92. DOI: https://doi.org/10.1080/11356405.2018.1559490

RAposo, Manuela y María Esther Martínez (2014), "Evaluación educativa utilizando rúbrica: un desafío para docentes y estudiantes universitarios", Educación y Educadores, vol. 17, núm. 3, pp. 499-513. DOI: https://doi. org/10.5294/edu.2014.17.3.6

SAIz Linares, Ángela y Teresa Susinos Rada (2018), "El practicum o cómo aprender a través de la reflexión colaborativa. El caso del problema de Elena”, Profesorado. Revista de Curriculum y Formación del Profesorado, vol. 22, núm. 1, pp. 393-411.

Salinas, Álvaro, Tamara Rozas y Pablo Cisternas (2018), "El foco y la profundidad de la reflexión docente en estudiantes de pedagogía 
en Chile", Perfiles Educativos, vol. 40, núm. 161, pp. 87-106.

Sснӧn, Donald (1998), El profesional reflexivo: cómo piensan los profesionales cuando actúan, Barcelona, Paidós.

Sewell, Allison, Sally Hansen y Kama Weir (2017), "Enhancing the Capabilities of Associate Teachers in the Practicum: A New Zealand case study", New Zealand Journal of Educational Studies, vol. 52, núm. 1, pp. 21-39.

STAKE, Robert E. (2010), Investigación con estudio de casos, Madrid, Morata.

Vygotsky, Lev S. (1979), Desarrollo de los procesos psicológicos superiores, Barcelona, Grijalbo-Crítica. 